\title{
Reprint
}

Max Wertheimer (1922)

\section{Untersuchungen zur Lehre von der Gestalt}

\section{Prinzipielle Bemerkungen}

Die wissenschaftliche Psychologie arbeitet fast überall, soweit sie konkrete Einzelforschung betreibt - bei aller Verschiedenheit der allgemeinen Lehrmeinungen -, mit einer sehr prägnanten Grundanschauung vom Psychischen; einer Anschauung, die in voller Konsequenz, in voller Wirklichkeit erfaßt, natürlich empfindenden Menschen immer von neuem recht eigentlich fremd, hölzern, ungeheuerlich erscheinen muß (obzwar sie mit sehr charakteristischen Faktoren moderner Weltanschauung aufs innerste zusammenhängt); die aber schon wegen ihrer unzweifelhaften Vorzüge im Sinn wissenschaftlicher Exaktheit gegenüber vageren Meinungen immer wieder mit Selbstverständlichkeit im konkreten Wissenschaftsbetriebe vorausgesetzt wird; - um so mehr, als man dazu neigt, manches Wesentliche dieser Grundanschauung als Erfordernis sauber wissenschaftlichen Verfahrens überhaupt anzusehen.

$\mathrm{Da}$ man z. B. bei der Aufgabe wissenschaftlicher Erfassung des Psychischen überall zunächst sauber die "Elemente" statuieren müsse, die der komplizierten Mannigfaltigkeit der psychischen Vorgänge im Nebeneinander zugrunde liegen und unter Verwendung allgemeiner sie betreffender Gesetzmäßigkeiten aus diesen Elementen dann durch Kombination, durch Und-Verbindung, zur richtigen Beschreibung und Erklärung der komplexen Vorgänge gelange -, das sind Thesen, die leicht als völlig selbstverständlich vorausgesetzt werden. Und: was auf solchem Boden an ernster Wissenschaftlichkeit, sauberer Exaktheit und Drängen zu konkreten Einzelentscheidungen erobert ist, soll nicht verloren gehen.

Aber: es ist in der Wissenschaft gut, auch Prinzipiellstes zu gegebener Zeit ernsthafter und konkreter Prüfung zu unterwerfen; nicht in nur allgemeinen, mehr spekulativen Erwägungen, sondern in konkretem Eindringen; im positiven Vorschreiten zu möglichst adäquater Erfassung des Gegebenen und im Vordringen zu Entscheidungsfragen innerhalb des Tatsächlichen.

Die Thesen jener Grundauffassung werden durch eine Reihe konkreter Befunde und Erfolge gestützt; manches, was sich in der Forschung ergab und immer mehr zu einer wirklichen Prüfung der prinzipiellen Grundlagen hätte hindrängen können, konnte doch schlecht und recht irgendwie eingegliedert werden oder wurde irgend mitgeschleppt; Gegenthesen, die extrem kontrastierend scheinen 
(oft mehr scheinen als sind), vermochten sich meist wegen ihres relativ vagen Charakters, wegen ihrer Unproduktivität im Sinn eines positiven Vordringens ins einzelne, wegen des Mangels der Gewinnung fruchtbarer Ergebnisse nicht wirklich durchzusetzen ( - und blieben auch oft, merkwürdig genug, beim selben Forscher in leerer Allgemeinheit unvermittelt neben dem andern).

Untersuchungen, über die im folgenden berichtet werden soll'1), führten immer klarer darauf, wie diese Grundauffassung - für manche Probleme im ersten Herangehen der Wissenschaft bewährt und tauglich -, vielfach doch nur in erster Annäherung aufrechterhalten werden könne; wie sie im Prinzip doch geradezu nicht richtig sein könne; daß es mit ad-hoc-Hinzufügung heterogener theoretischer Annahmen, mit Verwischungen und Verkleisterungen nicht getan sei; daß es im Prinzip schon anders liege, und in sehr konkreter Weise anders. (Es hatten sich Fragestellungen ergeben, so konkret, daß sie auch in speziellste Versuchsanordnungen eingriffen, und so prinzipiell, daß die Fundierung der psychischen Gegebenheiten und Vorgänge von Grund aus anders erschien.)

Hier sei zunächst einiges Prinzipielle in Form von Thesen gesagt; in der Art der Gegenüberstellung mag einiges Wesentliche klar werden. Es handelt sich hier um Klarstellung einer prinzipiellen Frage.

Für die oben gemeinte Grundauffassung sind $^{2}$ zwei einfach formulierbare Grundthesen charakteristisch:

Ich formuliere hier in manchem schärfer als es üblich ist; es soll sich hier nicht um irgendwelche "Lehrmeinungen" handeln, sondern um Sachliches in möglichster Prägnanz.

I. Die Mosaik- oder Bündelthese:

Allem "Komplexen" liegt zunächst, als Grundlage, die Summe nebeneinander gegebener elementarer Inhalte, Bestandstücke (Empfindungen usw.) zugrunde3. Man hat es im Grunde mit einer summativen Mannigfaltigkeit von verschiedenartigen Bestandstücken (einem "Bündel") zu tun'; alles weitere baut sich auf der Und-Summe der Elemente irgendweiter auf; zu Empfindungen treten etwa "Residuen" früherer Wahrnehmungen; treten Gefühle und allerlei Faktoren, wie "Aufmerksamkeitsvorgänge", Auffassungsvorgänge, Willensprozesse usw.

Auch das Gedächtnis knüpft sich an die Summe der Inhalte.

\footnotetext{
${ }^{1}$ Dem vorliegenden einleitenden Artikel soll eine Reihe spezieller Abhandlungen folgen. - Über das Wesentliche des hier Dargestellten habe ich seit 1912 vorerst hauptsächlich in akademischen Vorlesungen berichtet. (Vgl. auch "Über das Denken der Naturvölker", Zeitschr. f. Psych. 1911; "Exp. Studien über das Sehen von Bewegung”, Zeitsehr. f. Psych. 1912; “Über Schlußprozesse im produktiven Denken”, Berlin-Leipzig 1920.) Verweisen kann ich auf Untersuchungen von W. Köhler, Koffka, Gelb, Goldstein, Fuchs u. a.

2 In erster Linie in Hinsicht auf die Lehre von den Sinnestätigkeiten, der Wahrnehmung, den Vorstellungen und dem Denken.

3 Z. B. mit der konkreten Folge: habe ich $a_{1} b_{1} c_{1}$ und wird $b_{1}, c_{1}$ durch $b_{2} c_{2}$ ersetzt, so habe ich selbstverständlich $a_{i} b_{2} c_{2}$ (abgesehen nur von etwa additiv hinzukommenden Stückwirkungen).

${ }^{4}$ Abgesehen etwa von einer hoch darüber schwebenden, "nicht näher greifbaren" Einheit des Bewußtseins.
} 
II. Die Assoziationsthese:

Ist ein Inhalt a mit einem andern b öfter zusammen dagewesen, ("in raumzeitlicher Kontiguität"), so besteht die Tendenz, daß das Auftreten von a das Erscheinen von $b$ nach sich ziehe.

(Ist pum-lap öfter dagewesen und kommt nun einmal pum etwa in der Wahrnehmung, so fällt einem lap ein. So ist mein Freund mit seiner Telephonnummer assoziativ "verbunden”.)

(Das ist der einfache Sinn des Assoziationsgesetzes; unbeschadet der vielfach verschiedenen Formulierungsweise und Lehren; so ist es in konkreten Untersuchungen fundiert, so wird es in der Arbeit, in der konkreten Argumentation benutzt und so meint man es auch meist bei strenger wissenschaftlicher Verwendung. - Sagt man, wie es gelegentlich geschieht, statt "assoziiert" "einheitlich verknüpft", so verwischt man die Sachlage durch vage Konfundierung [meist ohne ernstliche Folgen].)

In der Assoziation ist eine bloße Existentialverbindung gegeben, eine Verbindung nur bezüglich des Auftretens der (irgendwelchen) Inhalte; eine Verkettung, die prinzipiell sachfremder Natur ist; die verketteten Inhalte sind gegeneinander beliebig; ihr inhaltliches Zueinander kommt prinzipiell nicht in Frage; wie sie zueinander stehen, spielt keine Rolle, sie haben keine innere Ingerenz aufeinander. Es führt keine Brücke prinzipiell von einem zum andern als die bloße Existentialverbindung 5 .

Und ebenso wie die Inhalte prinzipiell beliebig sind, sind es die aneinandergereihten Assoziationen selbst.

Wenn man diese These ernsthaft erfaßt, in aller Konsequenz, ohne Umfärbung durch angefügtes oder anklingendes Heterogenes, das Bild des Psychischen als Bündel von Inhalten, als Tummelplatz von Gewohnheit und Assoziation (und so i s $\mathrm{t}$ das theoretische Bild zu allermeist im Konkreten, trotz scheinbar oft entgegengesetzter "allgemeiner Lehrmeinung" -; mit allen charakteristischen Konsequenzen auch für Anwendungen z. B. in der Pädagogik, in der Erkenntnistheorie -), so kann es einem rätselhaft erscheinen, wieso - etwa im Materialismusstreit - im Gegensatz zur "blinden geistlosen Materie" auf das "Bewußtsein" so großer Wert gelegt wurde. Der Name Idealismus wirkt dann vielfach wie eine Erschleichung.

Auf die Formulierung dieser Thesen im einzelnen ist hier kein Gewicht gelegt; man kann, ohne das hier Wesentliche irgend zu tangieren, je nach der Lehrmeinung die Formulierungen ersetzen; es ist hier eine bequeme, dem Tatsächlichen des Wissenschaftsbetriebs naheliegende Form gewählt.

Beiden Thesen entspricht in einfacher Weise die übliche physiologische Vorstellung: die Summe der "erregten Zellen”, verbunden durch Leitungs-, durch "Assoziationsbahnen”; ein Aggregat nebeneinander funktionierender Apparate und Prozesse.

Prinzipiell identisch ist in den beiden Thesen - und darauf soll es hier ankommen das Und-Summenhafte: der Aufbau aus Stücken, die, das eine und das andere und ein drittes ... zunächst, primär, alles Weitere fundierend gegeben sind; in

\footnotetext{
${ }^{5}$ Entsprechend liegt es, wo "Ähnlichkeitsassoziation" auf der Grundlage der Teilgleichheit gemeint wird.
} 
Und-Verbindung; im Auch-Dasein; gegeneinander inhaltlich prinzipiell beliebig und ohne Ingerenz, es sei denn eine solche, die stückhaft von "unten her" - wieder von Stücken her - gemeint ist; entstehen darüber höhere Gebilde, Verbindungen, Komplexe, so bauen sich diese sekundär, von unten her, auf der Und-Summe der Stücke auf (wobei etwa wieder sachlich beliebig hinzutretende Funktionen, Akte, Verhaltungsweisen der Aufmerksamkeit usw. eine Rolle spielen).

W a s zusammengefügt erscheint, im Zugleich, im Nebeneinander, im Nacheinander, ist prinzipiell beliebig; für das Zusammensein ist der "Inhalt" oder das Zueinander von Inhalten eigentlich irrelevant. Keine sachlichen Momente sind für die Zusammengefügtheit bedingend, sondern inhaltsfremde, "sachäußere" Faktoren, wie z. B. das Oftzusammengewesensein, das simultane Beachten usw.

Es ist wie ein blindes Hantieren mit Bausteinen verschiedener Art; was zusammengefügt ist, wohin es führt, was geschieht, ist prägnant "kontingent".

Es ist das Bild des mechanischen Aneinanderfügens von Stücken einer Mannigfaltigkeit, die immer, in einfacher Summe zunächst das Eigentliche des Gegebenen bilden; in technischem Bilde für die erste These etwa: das Gesamtarbeitsprodukt einer Reihe nebeneinander gestellter einzelner Apparate ist gleich der Summe der irgendwelchen Arbeitsprodukte der einzelnen. Bezüglich der zweiten These - in Grobem - : gleichgültig was zugefügt wird, alles ist "gummiert"; Stücke beliebigster Art, wenn sie nur öfters äußerlich aneinandergedrückt sind oder werden -, haften aneinander, je öfter je mehr.

Kurz: die grundlegende Und-Summe, der Gang von unten nach oben, das Mechanische, das Beliebige, das sachlich Zufällige.

Den obigen Einzelthesen kann eine Reihe anderer - nicht bloß die Psychologie betreffende - an die Seite gesetzt werden. (Auch z. B. wie sich zeigen wird: Erkenntnis als "Rückführung auf Bekanntes" oder "Erklärungsbedürftig ist das Ungewohnte" und ähnliche, für die Wissenschaften nicht minder prinzipielle.) Die Thesen - und ebenso die im folgenden gegenübergestellten - sind durchaus nicht auf die Psychologie, nicht auf die Geisteswissenschaften beschränkt.

Es ist natürlich möglich, zu diesen Thesen $^{6}$ ) dort, wo sie nicht hinlangen, ergänzende Annahmen hinzutreten zu lassen, die dann oft eigentlich prinzipiell

\footnotetext{
${ }^{6}$ Man mag sich über die Schärfe dieser Bestimmungen vielleicht wundern; wo denn würde solches in solcher ungemilderter Präzisheit heute vertreten? Aber man besehe nicht so sehr die "allgemeinen Lehrmeinungen" sondern was einer tut; wie in konkreten Problemen argumentiert wird, wie gearbeitet wird, wie vorwärtsgegangen wird (wenn es sich um wirklich strenge Forschung handelt, die wir ja Gott sei Dank in der Psychologie hier und da haben); und was an konkretem positiven Gehalt hinter da verwendeten Terminis steckt. Im übrigen, hier kommt es auf prinzipielle Schärfung an; es handelt sich nicht um Meinungen, sondern um sachliche Fragen. In exakten Wissenschaften sollte man gewöhnt sein, gerade von dem Versuch strengen Vordringens in Hinsicht prinzipiellster Fragestellung - sofern diese nur wissenschaftlich wirklich ins Konkrete gerichtet, wissenschaftlich fruchtbar gerichtet ist - sich das Ernsthafteste zu erwarten. Es ist keine Frage, daß objektiv betrachtet die obigen Thesen in der konkreten psychologischen Arbeit in weitem Maß tatsächlich herrschen - schon mangels einer irgend an positiver Konkretheit vergleichbaren Methode - (man denke etwa an die Arbeiten über das Gedächtnis, an die Theorien vom Denken als Vorstellungsablauf, an das Hantieren mit "Residuen", an die Theorie der Alexie und Agraphie - um einige einfach liegende Fälle herauszugreifen). Und daß sie sich in manchen Punkten bewährt haben (wenn auch heute freilich viele an ihrer weiterführenden Fruchtbarkeit verzweifeln).
} 
anders gerichtet sind; die man aber ohne vermeintliche Tangierung der prinzipiellen Grundlagen ad hoc gebraucht.

(An diesen Thesen wird in dem hier Entscheidenden nichts wesentlich geändert durch die Annahme zur Summe der beliebigen einzelnen Stücke hinzutretender, an sie sich anschließender besonderer höherer Inhalte [wie in mancher charakteristischer Definition und manchem Gebrauch "Gestaltqualitäten", "Relationen" u. ähnl.]; oder durch Prozesse wie Einheitsstiftung durch "simultane Aufmerksamkeitsumfassung", oder durch sich anschließende ("beliebige") intellektuelle Akte, durch Hereinnahme von "Bedeutungsfunktion" im Sinn des "Zusammenhanges von Gegenstand und Name”, oder durch Konstatierung hinzutretender unanschaulicher Inhalte, wofern auch diese wieder stückhaft behandelt werden; oder durch additiv hinzutretende Willenstendenzen; oder durch einen Rekurs auf "Ganze und Teile", wofern "Ganze" doch wieder die Summe beliebiger Inhalte darstellen sollen).

Demgegenüber:

Nur selten, nur unter bestimmten charakteristischen Bedingungen, nur in sehr geringen Grenzen und vielleicht überhaupt nur in Annäherung liegt Und-Summenhaftigkeit wirklich vor; es erweist sich als nicht adäquat, diesen Grenzfall als typische Grundlage des Geschehens aufzufassen.

Nur selten: z. B. manchmal beim Schnupfen; im Zustand vollendeter Torheit; an charakteristischen Stellen innerhalb stockender Denkverläufe; bei Nebeneinandergegebenheit von kraß sachlich "Disparatem", gegeneinander sachlich Irrelevantem, das stückhafte Auffassung erzwingt (bei der Trennung, Abhebung zusammenhangloser Gestalten); unter Versuchsumständen, die durch "Einstellung" auf "Stück-Konstatierung", auf "Gestaltzerfall", auf Verflachung der Eindrücke hinwirken.

In sehr geringen Grenzen: Der "Umfang des Bewußtseins" ist für Stückhaftes außerordentlich gering; er ist dem Grade der Gestaltetheit funktional verbunden (was biologisch recht wichtig ist). Ähnlich bezüglich der Merkbarkeit, der Einprägsamkeit, dem Gedächtnis.

Vielleicht überhaupt nur in Annäherung: Auch wo man stückhafte Summativgegebenheit zunächst vermuten könnte, zeigt tieferdringende Deskription oft (und funktionale Gesetzmäßigkeiten bestätigen es), daß doch andres vorliegt; selbst in den eben genannten Beispielen - (auch der Eindruck des "Chaotischen" ist durchaus nicht einfach die Gegebenheit einer Und-Summe); daß oft nur eine typische Denkgewohnheit in der Wissenschaft zu der Annahme führte. Wirkliche

\footnotetext{
7 Man besehe die vorliegenden Definitionen; und sehe auch, wie in der konkreten Arbeit so oft diese "Inhalte" typisch als nur eben noch weitere, hinzukommende, undurchsichtige, unbehandelbare "Backsteine" fungieren oder als irgendwelche Produkte beliebiger Summen.
} 
Realisierung stückhafter Und-Gegebenheit ist nur in geringem Maße durchführbar; immer mit der Gefahr eines künstlichen Zustands, immer mit der Gefahr, die Gegebenheiten selbst zu ändern, zu verflachen, sie des Wesentlichsten zu entleeren.

Zur Adäquatheit: Für manche Probleme ist theoretisch summative Auffassung naheliegend; in mancher Beziehung gilt sie, oft in erster Annäherung (deshalb war es im ersten Herangehen der Wissenschaft für manche Probleme sehr tauglich, die stückhafte Auffassung zunächst zugrunde zu legen. Aber es soll vorwärts gegangen werden). Die Frage, ob sie als typische Grundlage aufzufassen sei, wird an bestimmten deskriptiven und funktionalen Tatbeständen prüfbar.

Das Gegebene ist an sich, in verschiedenem Grade "gestaltet": gegeben sind mehr oder weniger durchstrukturierte, mehr oder weniger bestimmte Ganze und Ganzprozesse, mit vielfach sehr konkreten Ganzeigenschaften, mit inneren Gesetzlichkeiten, charakteristischen Ganztendenzen, mit Ganzbedingtheiten für ihre Teile.

"Stücke" sind zu allermeist in konkreter Weise "als Teile" in Ganzvorgängen aufzufassen.

Die empirische Untersuchung zeigt nicht primären Aufbau aus Stücken, sondern Gradstufen von Gegebenheiten "in großen. Zügen" (in Hinsicht umfassender Ganzeigenschaften), in verschiedener Ausgeprägtheit, bis zu prägnant durchgestalteter Gegebenheit in Hinsicht aller "Unterganzer" und "Teile" "an ihrem Ort im Ganzen". Summatives Nebeneinander zweier Ganzer ist ein Spezialfall. - Konstatierung von "Teilen", stückhafte Auffassung (ob das nun höhere Unterganze sind oder sogenannte "Elemente") ist ein sehr realer, das Gegebene vielfach ändernder Prozeß; was "in einem Teil" gegeben ist, ist nicht prinzipiell unabhängig von anderem Gegebenen; Veränderungen von Teilen sind nicht prinzipiell ohne sachliche Ingerenz für die anderen, sondern haben solche oft, von klaren Ganzbedingungen her; es bestehen da gesetzliche funktionale Abhängigkeiten für das Geschehen (aus inneren "Strukturprinzipien"), indem die Teile nicht irgendwelche Stücke in primärer Und-Verbindung sind, sondern prägnant Teile in einem Ganzverlauf8.

Was zusammentritt, was "zusammengefaßt" erscheint, was "ergänzt" wird, ist nicht prinzipiell sachlich "beliebig" (und nicht prinzipiell von blinden sachfremden, "äußeren" Faktoren bestimmt, wie z. B. stückhafter Gewohnheit), sondern vielfach von konkreten Gestaltgesetzen her bedingt. Es zeigen sich Tendenzen zu bestimmter "ausgezeichneter Gestalt", Ganzgerichtetheiten, Gesetzmäßigkeiten,

\footnotetext{
${ }^{8}$ Auch bezüglich der ganzen Menschen. - Man bedenke von hier aus auch die Konsequenzen für die Probleme des "psychologischen Realismus".

9 In welcher "Beliebigkeit" man merkwürdigerweise vielfach geradezu eine besondere Würde des Psychischen sieht. - Freie Beliebigkeit ist nur ein Grenzfall; und kein grundlegend typischer.
} 
bei denen von sachlichen Ganzbedingungen her sich Teile "aus innerer Notwendigkeit bestimmen", "gefordert werden".

Nicht also sind "die Stücke" zunächst als das "prius" anzusetzen, als Fundament in Und-Verbindung und unter prinzipiell sachfremden Bedingungen ihres Auftretens, sie stehen vielfach als Teile unter sachlichen Bedingtheiten von ihrem Ganzen her, sind von ihnen her "als Teile" zu verstehen. [Wobei freilich "Ganze" und "Teile" in einem sehr prägnanten Sinn gemeint sind, der im weiteren konkret werden soll ${ }^{10}$.]

Nicht also sind "Gestalten" hier "zur Summe hinzukommende Inhalte", auf primär gegebenen Stücken sich "subjektiv aufbauende", kontingente, "nur subjektiv bedingte", "beliebige" Gebilde; nicht einfach blinde, weitere "Qualitäten”, im Grunde ebenso stückhaft und unbehandelbar wie die "Elemente"; nicht bloß etwas "zu einem Material hinzukommendes", "bloß Formales"; sondern es handelt sich um Ganze und Ganzprozesse mit vielfach sehr bestimmten inneren, sachlichen Gesetzlichkeiten, um Strukturen mit konkreten Strukturprinzipien. Auch die sogenannten "Elemente" sind als Teile anzusetzen oder als Derivate.

$\mathrm{Daß}$ von diesen Thesen aus sich auch bestimmte Zugänge eröffnen zu Problemen ganz anderer Relevanz als Wahrnehmung und Gedächtnis -, zu Problemgebieten psychischer und geistiger Werte, ist leicht zu sehen; ebenso, daß in der allgemeinen Fragestellung beträchtliche philosophische Konsequenzen liegen.

Manche theoretische Denkgewohnheit in der Wissenschaft mag dem Gesagten entgegenstehen und das Verständnis in dieser vorläufig abstrakten Formulierung erschweren; man mag im folgenden sehen, daß das Gesagte sich vielfach in sehr einfacher, greifbarer Weise konkretisiert. - Die natürliche Denkweise des lebendig empfindenden Menschen steht dem Gesagten viel näher als den Thesen der ersten Grundauffassung.

Einiges im Sinn dieser Thesen sei hier gleich kurz erwähnt :

1. Bei der wissenschaftlichen Behandlung der Wahrnehmung ist hiernach nicht fundierend auszugehen von der "Summe" der Einzelreize einerseits und der "Summe" der Empfindungen andererseits in Einzelentsprechung unter sekundärer summativer Hinzufügung weiterer Faktoren, sondern - und das ist schlicht tatsachennäher - von der Reizkonstellation einerseits und dem psychisch tatsächlich Gegebenen in seinem Gestalthaften andererseits ${ }^{11}$. Neben den Faktoren der Reizkonstellation (deren Ganzfaktoren schon zu berücksichtigen sind) sind gesetzliche subjektive Faktoren ${ }^{12}$ bestimmend, welche in wesentlicher Hinsicht charakteristische Ganzbedingtheiten darstellen.

\footnotetext{
${ }^{10}$ Keineswegs handelt es sich um den Gegensatz von "Analyse" und "Synthese" in der oft üblichen flachen Denkweise dieses Gegensatzes: weder geht es adäquat im Und-Zusammenhänge "von unten nach oben”, noch in subtraktiver Abstraktion stückhaft "von oben nach unten".

${ }^{11}$ Die Befürchtung, daß damit aller Systematik fester Boden schwinde, daß eine unbeherrschbare Vielfältigkeit wissenschaftliche Behandlung unmöglich mache, behebt sich bald: in dem Vordringen zu einfachen Gestaltgesetzen.

${ }^{12}$ Es ist nicht so, daß die Seele mit dem von den Reizen gelieferten "hinsichtlich der Gestaltung" "frei Beliebiges" machen kann oder gar macht.
} 
Den obigen Thesen entspricht eine bestimmte physiologische Vorstellung; nicht komme es im Gegensatz zur üblichen Behandlungsweise grundlegend an auf eine "Summe" von Erregungen einzelner Stellen, verbunden durch bloße Leitungsassoziation, sondern auf charakteristische physiologische Gesamtprozesse, spezifische Ganzvorgänge. (Die erregten Zellen sind Teile eines lebendigen Ganzen, stehen in bestimmten funktionellen Zusammenhängen, ihre Prozesse sind als Teilprozesse größerer Ganzprozesse anzusehen.) Siehe W. Köhler: "Die physischen Gestalten”. Vieweg 1920. - In mancher Hinsicht handelt es sich hier - wie Köhler zeigte - im Grunde um Anwendung modern-physikalischer Denkweisen auf die physiologischen Probleme (Denkweisen, die in der modernen Physik allenthalben durchgedrungen sind; freilich in der Arbeit, nicht so schon in der erkenntnistheoretischen Spiegelung); in mancher Hinsicht um Sachverhalte, die im Sinne der Thesen klar in der modernen Physik aufweisbar sind.

Den Kern des Gesagten gaben die allgemeinen Sätze am Schluß der "Experimentellen Studien über das Sehen von Bewegung” (Zeitschr. f. Psych. 1912, S. 91-92 besonders Anm. 3), in bloß physiologischer Ausdrucksweise; in nur physiologischer wegen der großen Belastung der üblichen psychologischen und erkenntnistheoretischen Begriffe. (So ließ sich's prägnant und kurz sagen.) Das wurde merkwürdigerweise von einzelnen Forschern dahin mißverstanden, daß allgemein im Gegensatz zu einer "psychologischen" Theorie, unter "Verzicht auf eine solche" bloß physiologische Behandlungsweise gesetzt werden sollte. Ähnlich wurde von manchen ein Satz eines Forschers bei Darstellung der obigen Theorie (Koffka, Beiträge zur Psychologie der Gestalt. Barth. 1919. S. 250) mißverstanden, als ob er besagte, daß es auf eine "psychologische Analyse" nicht ankommen könne wobei hier für das Allgemeine nur die übliche, stückhafte (rein subtraktiv - abstraktive) psychologische Analyse streng abgelehnt war. - Ganz im Gegensatz: ein gestaltmäßiges, strukturgemäßes Eindringen, ein psychologisch vorwärtsdringendes Erfassen und Begreifen des Geschehens wird im Sinn obiger Thesen gefordert, ja in wesentlicher Hinsicht erst ermöglicht.

2. Gänzlich abgesehen von Reizbedingungen und physiologischen Faktoren, rein innerhalb des Psychologischen: das theoretische Vorschreiten "von unten nach oben" wäre nicht prinzipiell das adäquate, sondern vielfach ist der Weg "von oben nach unten" gefordert: das Erfassen bestimmter Ganzeigenschaften, Ganzbedingungen, Struktureigenschaften und von da aus der Weg zu "Teilen" im prägnanten Sinn dieses Wortes. Es ist ein folgenreicher Unterschied, ob ich sage: es ist $a$ da und $b$ und $c . .$. - Inhalte für sich (etwa jeder durch seinen Reiz bedingt, oder von stückhaften Reproduktionsgesetzen her) und diese Und-Gegebenheit der Summe als die Grundlage ansehe, an die eventuell sich Weiteres knüpfen mag - oder ob ich sage: ich habe diese und jene durch konkrete Charaktereigenschaften und Gesetzlichkeiten bestimmte Ganze und Ganzverläufe, aus denen ich durch Zerstückung, durch Realteilung (was man als bloßen Wechsel der Aufmerksamkeit ansah oder als glatt subtraktive Abstraktion u. ähnl.) Teile gewinnen kann, - Derivate -, Unterganze zunächst; von denen ich aber unter diesen neuen Bedingungen dann freilich nicht mit Sicherheit weiß, ob sie auch ebenso als Teile in dem Ganzen waren; ja von denen ich unter Umständen klar feststellen kann, wiefern dieser Vorgang gesetzlich Änderungen bedingt. Und ebenso beim Zusammentreten von Inhalten zu größeren Ganzen.

3. Besonders folgenreich werden Befunde, die dahin weisen, daß, was sich natürlicherweise verbindet (und ebenso, was sich $\mathrm{zu}$ trennen sucht), ja, was zu gegebenem Psychischen - etwa als Ergänzung - hinzutritt, nicht prinzipiell sachlich beliebig ist, nicht prinzipiell durch äußere, inhaltsfremde Faktoren bedingt ist (wie Gewohnheit und Erfahrung im rein stückhaften Sinn dieser Worte), sondern durch sachliche Ganzfaktoren, durch konkrete Gestaltgesetzlichkeiten. 
Merkvorgänge selbst enthalten wesentlich Gestaltprozesse; das Gedächtnis knüpft sich in erster Linie an Ganzeigenschaften und Strukturzusammenhänge; das Wesentliche der Gedächtnisprozesse (oder auch der "Erfahrung") erschöpft sich nicht in der Anknüpfung an Summe und Folge, nicht in der an Ganze, wofern diese im Grund bloß summative Stückganze sein sollen. Assoziation, Gewohnheit im Sinne inhaltsbeliebiger Existenzialverbindung (das mechanische Gedächtnis überhaupt) ist bloß Grenzfall.

Denkvorgänge, Vorgänge bei originärer Lösung eines Problems, Vorgänge beim Erfassen und Begreifen (im prägnanten Sinn dieser Worte, etwa im grundlegenden Übergang vom Unbegriffenen zum Begriffenen), Vorgänge beim Sehen eines Problems - lösen sich ab von bloßen Gedächtnisvorgängen, mit denen sie "als Vorstellungsablauf” unrettbar verquickt schienen; lösen sich ab von Vorgängen im Sinn stückhafter Generalisation, stückhaft subtraktiver Abstraktion, Kombination usw.; erweisen sich in ihrem Wesentlichen als konkret-charakteristische, bestimmt geartete Gestaltprozesse ${ }^{13}$ (die ihre Analogien einerseits in Wahrnehmungsprozessen finden, andererseits in Gefühls-, in Willensvorgängen).

Das wäre, freilich in kurzer Andeutung, eine Reihe von Thesen; es sind in ihnen mehrfach verschiedene, voneinander nicht unbedingt abhängige Behauptungen enthalten. Es kann nun nicht etwa auf theoretische Diskussion in vager Allgemeinheit ankommen; alles kommt darauf an, in vorsichtigster Weise, in wissenschaftlicher Exaktheit, in strenger Tatsachentreue zu konkreten Ergebnissen und zu Entscheidungen vorzudringen ${ }^{14}$.

Es mag aber hier noch eine besondere Konsequenz angedeutet werden, die von umfassender Bedeutung ist.

Die "Ganzbedingungen", die hier mehrfach erwähnt wurden, sind für die Wissenschaft durch konkrete Forschung zu gewinnen. Es eröffnet sich aber aus ihnen eine Möglichkeit, auf welche manches positive Ergebnis klar hinweist: treten an Stelle von Vorgängen nach bloß äußeren inhaltsfremden Faktoren Vorgänge, die sich durch "innere Gesetze vom Ganzen" her bestimmen, so ist nicht mehr, was zusammentritt, was ergänzt wird, was geschieht, im Grunde sinnlos, blind, beliebig, mechanisch, durch Gewohnheit bedingt ...; an Stelle von prägnant

\footnotetext{
${ }^{13} \mathrm{Vgl}$. Schlußprozesse im produkt. Denken. S. $12 \mathrm{f}$.

${ }^{14}$ Ich gehe hier nicht auf Literaturangaben ein; wie sehr manches in theoretischen Entwicklungen besonders der letzten Jahre (in der Psychologie z. B. in der Theorie der Gestaltqualitäten, Komplexqualitäten, fundierten Inhalte, Gebilde usw.) in ähnliche Richtung drängt, ist wohl leicht zu sehen; wenn es dabei vielfach doch nur zu einem bloßen Meinungsdurcheinander gekommen ist und zu Unfruchtbarkeit der Ansätze, so liegt das wohl einfach daran, daß das prinzipielle Positive nicht entscheidend gepackt wurde. - Besieht man von den obigen Thesen aus die vorliegende psychologische Literatur, die konkreten Ergebnisse der Einzelforschungen, so finden sich allenthalben Einzelbefunde, die schon in allerlei Hinsichten im Sinne dieser Thesen heranzuziehen sind (die zum Teil, weil sie nicht prinzipiell gesehen wurden, ohne Konsequenzen blieben).
} 
sinnlosen oder recht eigentlich nur zufällig sinnhaften Vorgängen ermöglichen sich echt sinnvolle.

Man kann sagen:

In einem gewissen Sinn kann nicht echt Sinnvolles erreicht werden durch Ausgehen "von unten nach oben".

Man mag hier - vorläufig - sinnvoll in der sehr charakteristischen Bedeutung dieses Wortes im täglichen Leben verstehen; bei dem Erfassen eines inneren Zusammenhangs, dem Spüren einer inneren Notwendigkeit, bei sinnvoller Voraussage, sinnvoller Ergänzung, sinnvollem Verhalten; wo das Geschehende nicht durch blinde äußere Faktoren sich bestimmt, sondern durch sachliche "innere Gefordertheit" ${ }^{\prime \prime}$.

\begin{abstract}
Immerhin mag schon hier eine Definition angedeutet werden: im Idealfall sinnvoll ist ein Ganzes dann, wenn "sachliches Zueinandergehören" entscheidend wird: wenn Teile nicht als irgendwelche an irgendwelchen Stellen im Ganzen stehen, sondern in ihrem Sein und So-Sein an dieser ihrer Stelle von einem nicht teilsummativen Strukturprinzip ihres Ganzen gefordert werden. Man sieht, eine bestimmte Theorie des Verhältnisses von Ganzen und Teilen liegt hier zugrunde. Die einzelnen Termini können erst später ihre Konkretisierung finden; sie betreffen vielfach schlicht greifbare Momente an Sachverhalten. - Im übrigen ist die in der Definition verwendete Bestimmung zwar notwendig, aber nicht erschöpfend; zur vollen Definition gehören noch weitere Bestimmungen.
\end{abstract}

Von der Mosaik- und Assoziationsthese aus ist Erfassung, Erklärung, irgend sinnvoll, sachgemäßen Geschehens oder Seins als solchem prinzipiell nicht erreichbar; $\mathrm{n} \mathrm{u} \mathrm{r}$ in indirekter Weise, als Zufallseffekt. (Wie etwa im Schrotflintenprinzip, in der assoziativen Herleitung eines Denkverlaufs usw.)

Denn: für sie ist konstitutiv das Und-Summenhafte, sachlich gegenseitig Blinde der Stücke; der Aufbau aus Stücken von unten her und zwar so, daß inhaltsfremde, äußere Faktoren bestimmen, was verbunden wird; während für sinnvolle Vorgänge charakteristisch sein soll, daß, was geschieht, aus sachlichen Gefordertheiten geschieht, aus inneren Gründen.

Gäbe es so etwas tatsächlich nicht, so müßte man sich damit abfinden; aber das ist zunächst eine Tatsachenfrage. (Das scheint eine Grundfrage der Wissenschaft; inwieweit, inwiefern das Seiende sinnvoll sei; und man wird sie wohl beantworten müssen: Es gibt Sinnvolles und - es gibt [wenigstens zunächst] - Sinnloses; Lückenhaftes, blind Verkrüppeltes, von ungefähr aneinander Geratenes, aneinander Gekettetes, belanglos Aggregiertes.)

\footnotetext{
${ }^{15}$ Hume hat bei seinen folgenreichen Beispielen nur einen Typus von Fällen ins Feld geführt - bezüglich welcher er auch recht haben mag - man setze aber seinem Fall der Voraussage des Fallens eines loszulassenden Körpers etwa gegenüber den Fall der Voraussage eines kleinen Teils einer sonst gegebenen Fallkurve - und sieht bald, daß der Prozeß sich hier nicht einfach auf gewohnte Verkettung irgendwelcher, gegeneinander inhaltlich beliebiger Inhalte gründet, sondern daß es sich hier um "sachliche", "innere" Motive handelt; und nicht nur in psychologischer Hinsicht. (Auch Naturhaftes steht in Frage.)
} 
Auch im Effekt Sinnvolles kann eigentlich sinnlos sein; wenn nämlich das Taugliche, das faktisch Sinngemäße geschieht, nicht weil es das Taugliche ist, nicht aus innerem Prinzip des Ganzen, sondern aus äußerem Grund: zufällig oder gesetzlich, aus Drill, Assoziation, Gewohnheit. (Oder aus allgemeinen Gesetzen, wofern diese im Grunde wieder nichts als eine blinde Und-Summation zwischen Stückhaftem darstellen.)

[Viel wurde hierbei versperrt durch eine kurzsichtige Meinung, die hier fordern würde: "Wenn das Taugliche geschieht, ohne daß der Handelnde weiß, daß es das Taugliche sei und es also ohne bewußte Einsicht tut"; aber das ist mindest zu eng gedacht: Die obige Formulierung bezieht sich durchaus nicht auf Bewußtseinhaftes allein oder auch nur in erster Linie; sondern ist sehr viel allgemeiner.]

Ein äußeres wissenschaftliches Kriterium für das Zufallshafte, Unechte und im Grunde Unzureichende stückhafter Erklärungen tatsächlich sinnvollen Geschehens ergibt sich beispielsweise in der Prüfung der Variabilität: Aus Mosaik- und Assoziationsthese folgen bestimmte Bedingungen für das Geschehen bei Variation der Sachlage, die eine Prüfung ermöglichen.

Bezüglich einer Verhaltungsweise (schon z. B. einer bei bestimmter Sachlage dagewesener und gemerkter oder gelernter Reaktion) müßte bei neuer Variation der Sachlage das Geschehen im Prinzip von der summativen Ähnlichkeit der Bedingungen abhängen. Bei einer summativ sehr geringen Änderung der Umstände - die die alte Reaktion schon gänzlich sinnlos machen kann - muß diese prinzipiell eher erwartet werden als bei einer summativ sehr viel stärkeren Änderung, die alle Stücke verändert haben kann, bei der aber das, worauf es im ganzen ankam, erhalten geblieben ist; (es sei denn, daß ein Surrogaterfolg nicht schon durch bestimmte Bedingungen ermöglicht wurde, z. B. eine große Zahl von Variationen eingedrillt ist, so, daß die Häufung mechanisch zu bestimmten "allgemeinen" Verhaltungsweisen führen konnte u. ähnl.). - Bei sachlichtauglichem Material können so Entscheidungsfragen gestellt werden.

Anmerkung der Herausgeber: Rechtschreibung und Grammatik wurden beibehalten, ebenso die Gliederung in verschiedene Schriftgrößen und -arten. Verändert wurde die Nummerierung der Fußnoten, die hier, bedingt durch veränderte Seitenumbrüche, im Gegensatz zum Original durchlaufend gezählt werden. 